\title{
A NONCONFORMING PRIMAL MIXED FINITE ELEMENT METHOD FOR THE STOKES EQUATIONS
}

\author{
Sungmin Cho And Eun-Jae Park
}

\begin{abstract}
In this article, we propose and analyze a new nonconforming primal mixed finite element method for the stationary Stokes equations. The approximation is based on the pseudostress-velocity formulation. The incompressibility condition is used to eliminate the pressure variable in terms of trace-free pseudostress. The pressure is then computed from a simple post-processing technique. Unique solvability and optimal convergence are proved. Numerical examples are given to illustrate the performance of the method.
\end{abstract}

\section{Introduction}

Basic mathematical models in fluid and solid mechanics are expressed in terms of a set of partial differential equations with the physical unknowns such as pressure, velocity, stress, and/or an appropriate energy variable. For example, the original physical equations for incompressible Newtonian flows induced by the conservation of momentum and the constitutive law are given by the stress-velocity-pressure formulation [18]. The name and many of the original concepts for the mixed methods are originated in solid mechanics where it was desirable to have simultaneous approximations of certain quantities of interest. For the Stokes equations governing flows of incompressible viscous fluids, Galerkin mixed methods based on the pressure-velocity formation are wellunderstood [19]. The pseudostress-displacement formulation was proposed by Arnold and Falk [2] for the equations of linear elasticity which does not require symmetric tensors and hence facilitates the use of Raviart-Thomas mixed finite elements developed for scalar second order elliptic equations. The pseudostressvelocity formulation is then exploited to study first-order div least-squares finite element methods for the Stokes system by Cai, Lee, and Wang [12].

Received September 9, 2013.

2010 Mathematics Subject Classification. Primary 58B34, 58J42, 81T75.

Key words and phrases. primal mixed finite elements, nonconforming methods, error estimates, Stokes problems, pseudostress-velocity formulation.

This research was supported by Basic Science Research Program through the National Research Foundation of Korea (NRF) funded by the Ministry of Education, Science and Technology NRF-2012R1A2A2A01046471. 
Primal mixed finite element methods have been studied for diffusion and heat equations $[3,4,11,14,20,23,24]$. The method gains popularity thanks to the following features. First, it has a local mass conservation property and conserves fluxes node-wise. Nodal fluxes are of interest in some applications. For example, nodal fluxes are related with nodal forces in structural mechanics. Moreover, the primal mixed finite element method can resolve layer structures for solutions to partial differential equations such as semiconductor device equations, especially drift-diffusion equations [11, 23]. Second, the primal mixed method provides a softening of the quadratic form associated with the principal part of model equations and it is a starting point of study of the method of enhanced assumed strains, which is popular in computational mechanics $[6,7,8,25,26]$.

For applications to computational fluid dynamics, finite element methods of nonconforming type are attractive since they easily fulfil the discrete version of the inf-sup condition $[17,19]$. Another advantage of nonconforming finite elements is that the unknowns are associated with the element faces so that each degree of freedom belongs to at most two elements. Various applications of dual/primal mixed finite element methods can be found in $[1,10,15,19,21,24]$. In this paper, we use the incompressibility condition to eliminate the pressure variable from the Stokes system which yields the traceless pseudostress condition. We propose and analyze a nonconforming primal mixed finite element method for the velocity and pseudostress formulation of the Stokes system. The pressure is then computed from a simple post-processing technique.

The organization of the remainder of the paper is as follows. In the next section, we explain the pseudostress-velocity formulation for the Stokes problem and prove unique solvability of the variational problem appropriate for primal mixed method. In Section 3, a nonconforming primal mixed procedure is introduced. In Section 4, we derive optimal order error estimates in $L^{2}$-norm on the triangular mesh. Numerical results are given in the last section which supports our theory developed in this paper.

We finish this section with some notations used in this paper. Let $\mathbb{R}^{2}$ be the field of two-dimensional vector space and denote the field of $2 \times 2$ matrix functions $\mathbb{M}_{2}$. Throughout the paper, we adopt the convention that a bold Greek character denotes a matrix in $\mathbb{M}_{2}$ and a bold Latin character in lower case denotes a vector in $\mathbb{R}^{2}$. For a vector function $\mathbf{v}=\left(v_{1}, v_{2}\right)^{t} \in \mathbb{R}^{2}$, we define

$$
\nabla \mathbf{v}=\left(\begin{array}{ll}
\frac{\partial v_{1}}{\partial x} & \frac{\partial v_{1}}{\partial y} \\
\frac{\partial v_{2}}{\partial x} & \frac{\partial v_{2}}{\partial y}
\end{array}\right), \quad \operatorname{div} \mathbf{v}=\frac{\partial v_{1}}{\partial x}+\frac{\partial v_{2}}{\partial y}
$$

and for a tensor function $\boldsymbol{\tau}=\left(\tau_{i j}\right)_{1 \leq i, j \leq 2} \in \mathbb{M}_{2}$, we define

$$
\operatorname{div} \boldsymbol{\tau}=\left(\begin{array}{c}
\frac{\partial \tau_{11}}{\partial x}+\frac{\partial \tau_{12}}{\partial y} \\
\frac{\partial \tau_{21}}{\partial x}+\frac{\partial \tau_{22}}{\partial y}
\end{array}\right), \quad \operatorname{tr} \boldsymbol{\tau}=\sum_{i=1}^{2} \tau_{i i}
$$


The inner-product between vectors and the Frobenius inner-product between tensors are defined, respectively, by

$$
\mathbf{u} \cdot \mathbf{v}=u_{1} v_{1}+u_{2} v_{2} \text { and } \boldsymbol{\sigma}: \boldsymbol{\tau}=\sum_{1 \leq i, j \leq 2} \sigma_{i j} \tau_{i j} .
$$

We use the standard Sobolev spaces $H^{s}(\omega)$ and $H_{0}^{s}(\omega)$ for $s \geq 0$ with associated norm $\|\cdot\|_{s, \omega}$. We let $(\cdot, \cdot)_{\omega}$ denote the $L^{2}(\omega)$ inner product. In the case $\omega=\Omega$ the lower index is dropped, e.g., $\|\cdot\|_{s, \Omega}=\|\cdot\|_{s}$ and $(\cdot, \cdot)_{\Omega}=(\cdot, \cdot)$. When $s=0$, we simply write $\|\cdot\|_{0, \Omega}=\|\cdot\|$. We define $H^{-s}(\omega):=\left(H_{0}^{s}(\omega)\right)^{*}$ as the dual space of $H_{0}^{s}(\omega)$. Extending the definitions to vector- and matrixvalued functions, we let $H^{s}\left(\omega, \mathbb{R}^{2}\right)$ (or simply $\left[H^{s}(\omega)\right]^{2}$ ) and $H^{s}\left(\omega, \mathbb{M}_{2}\right.$ ) (or $\left.\left[H^{s}(\omega)\right]^{2 \times 2}\right)$ denote the Sobolev spaces over the set of 2 -dimensional vectorand $2 \times 2$ matrix-valued functions, respectively. We still use $(\cdot, \cdot)_{\omega}$ to denote the $L^{2}\left(\omega, \mathbb{R}^{2}\right)$ and $L^{2}\left(\omega, \mathbb{M}_{2}\right)$ inner product. Also, $\|\mathbf{v}\|_{L^{2}(\omega)}^{2}:=(\mathbf{v}, \mathbf{v})_{\omega}=\int_{\omega} \mathbf{v} \cdot \mathbf{v} d x$ and $\|\boldsymbol{\tau}\|_{L^{2}\left(\omega, \mathbb{M}_{2}\right)}^{2}:=(\boldsymbol{\tau}, \boldsymbol{\tau})_{\omega}=\int_{\omega} \boldsymbol{\tau}: \boldsymbol{\tau} d x$.

\section{Pseudostress-velocity formulation}

Let $\Omega$ be a bounded polygonal domain in $\mathbb{R}^{2}$ with smooth boundary $\partial \Omega$. Consider the Stokes equations:

$$
\left\{\begin{aligned}
-\nu \triangle \mathbf{u}+\nabla p=\mathbf{f} & \text { in } \Omega, \\
\operatorname{div} \mathbf{u}=0 & \text { in } \Omega, \\
\mathbf{u}=0 & \text { on } \partial \Omega,
\end{aligned}\right.
$$

where $\mathbf{f}=\left(f_{1}, f_{2}\right)^{t}$ is the external body force, $\nu$ is the kinematic viscosity of the fluid, and $\mathbf{u}=\left(u_{1}, u_{2}\right)^{t}$ and $p$ are the velocity vector and pressure, respectively.

To obtain the pseudostress-velocity formulation of (1), introduce a new independent pseudostress tensor

$$
\boldsymbol{\sigma}=\nu \nabla \mathbf{u}-p I
$$

where $I$ is a $2 \times 2$ identity matrix. Taking trace of (2) and using the divergence free condition give

$$
p=-\frac{1}{2} \operatorname{tr} \sigma
$$

Also let $\mathcal{A}: \mathbb{M}_{2} \rightarrow \mathbb{M}_{2}$ be the deviatoric operator

$$
\mathcal{A} \boldsymbol{\tau}=\boldsymbol{\tau}-\frac{1}{2} \operatorname{tr}(\boldsymbol{\tau}) I \text { for all } \boldsymbol{\tau} \in \mathbb{M}_{2} .
$$

We immediately notice that $\mathcal{A}$ is a projection onto the trace-free subspace of $\mathbb{M}_{2}$ and

$$
\operatorname{ker}(\mathcal{A})=\{f I \mid f \text { is a scalar function }\} .
$$

Also, we can easily show that the following properties of the operator $\mathcal{A}$ hold,

$$
\begin{aligned}
(\mathcal{A} \boldsymbol{\tau}, \boldsymbol{\sigma}) & =(\boldsymbol{\tau}, \mathcal{A} \boldsymbol{\sigma}), \\
(\mathcal{A} \boldsymbol{\tau}, \mathcal{A} \boldsymbol{\tau}) & =(\mathcal{A} \boldsymbol{\tau}, \boldsymbol{\tau})
\end{aligned}
$$




$$
\begin{aligned}
& =(\boldsymbol{\tau}, \boldsymbol{\tau})-\frac{1}{2}(\operatorname{tr} \boldsymbol{\tau}, \operatorname{tr} \boldsymbol{\tau}), \\
\|\mathcal{A} \boldsymbol{\tau}\| & \leq\|\boldsymbol{\tau}\| .
\end{aligned}
$$

Then, from (2), (3), and (4), we arrive at $\kappa \mathcal{A} \boldsymbol{\sigma}=\nabla \mathbf{u}$ where $\kappa$ is $\frac{1}{\nu}$. Thus, by the definition of the pseudostress $\sigma$, which is not necessarily symmetric, system (1) can be rewritten as:

$$
\left\{\begin{aligned}
\kappa \mathcal{A} \boldsymbol{\sigma}-\nabla \mathbf{u}=0 & \text { in } \Omega, \\
-\operatorname{div} \boldsymbol{\sigma}=\mathbf{f} & \text { in } \Omega,
\end{aligned}\right.
$$

with the same boundary condition. It is well-known that the stationary Stokes equation has a unique solution provided that the average condition

$$
\int_{\Omega} p d x=0
$$

which, together with (3), implies the trace-free condition

$$
\int_{\Omega} \operatorname{tr} \sigma d x=0
$$

With the function spaces

$$
\mathbf{L}=\left\{\boldsymbol{\tau} \in\left[L^{2}(\Omega)\right]^{2 \times 2}: \int_{\Omega} \operatorname{tr} \boldsymbol{\tau} d x=0\right\} \text { and } \mathbf{V}=\left\{\mathbf{v} \in\left[H_{0}^{1}(\Omega)\right]^{2}\right\},
$$

we arrive at the following primal variational formulation for the equation (6): For given $\mathbf{f} \in\left[L^{2}(\Omega)\right]^{2}$, find $\{\boldsymbol{\sigma}, \mathbf{u}\} \in \mathbf{L} \times \mathbf{V}$ such that

$$
\left\{\begin{aligned}
(\kappa \mathcal{A} \boldsymbol{\sigma}, \boldsymbol{\tau})_{\Omega}-(\boldsymbol{\tau}, \nabla \mathbf{u})_{\Omega} & =0 & & \forall \boldsymbol{\tau} \in \mathbf{L} \\
(\boldsymbol{\sigma}, \nabla \mathbf{v})_{\Omega} & =(\mathbf{f}, \mathbf{v}) & & \forall \mathbf{v} \in \mathbf{V} .
\end{aligned}\right.
$$

We will find the following lemmas useful in proving the existence and uniqueness of problem (7). First, we have from integration by parts:

Lemma 2.1. Let $\mathbf{v} \in \mathbf{V}$. Then we have

$$
\begin{gathered}
\int_{\Omega} \nabla \mathbf{v} d x=0, \\
\int_{\Omega} \operatorname{tr}(\nabla \mathbf{v}) d x=\int_{\Omega} \operatorname{div} \mathbf{v} d x=0 .
\end{gathered}
$$

The following is well-known (see, for example, [10], [13]).

Lemma 2.2. For any $\boldsymbol{\tau} \in \mathbf{L}$, we have

$$
\|\boldsymbol{\tau}\|_{0, \Omega} \lesssim\|\mathcal{A} \boldsymbol{\tau}\|_{0, \Omega}+\|\operatorname{div} \boldsymbol{\tau}\|_{0, \Omega} .
$$

Here and throughout the paper, we use the notation $X \lesssim Y$ which means that there exists a generic constant $C$ independent of the mesh parameter $h$ such that

$$
X \leq C Y .
$$

Theorem 2.3. The variational problem (7) has a unique solution. 
Proof. Define the bilinear form $b: \mathbf{L} \times \mathbf{V} \rightarrow \mathbb{R}$ by

$$
b(\boldsymbol{\tau}, \mathbf{v})=(\boldsymbol{\tau}, \nabla \mathbf{v})_{\Omega} .
$$

By Lemma 2.1, for any $\mathbf{v} \in \mathbf{V}$, we have $\nabla \mathbf{v} \in \mathbf{L}$ and

$$
\sup _{\boldsymbol{\tau} \in \mathbf{L}} \frac{b(\boldsymbol{\tau}, \mathbf{v})}{\|\boldsymbol{\tau}\|_{0}} \geq \frac{b(\nabla \mathbf{v}, \mathbf{v})}{\|\nabla \mathbf{v}\|_{0}}=\|\nabla \mathbf{v}\|_{0} \gtrsim\|\mathbf{v}\|_{1} .
$$

Define the bilinear form $a:\left[L^{2}(\Omega)\right]^{2 \times 2} \times\left[L^{2}(\Omega)\right]^{2 \times 2} \rightarrow \mathbb{R}$ by

$$
(\boldsymbol{\sigma}, \boldsymbol{\tau})_{\Omega} \mapsto(\kappa \mathcal{A} \boldsymbol{\sigma}, \boldsymbol{\tau})_{\Omega}
$$

Let $B: \mathbf{L} \rightarrow \mathbf{V}^{\prime}$ be a linear continuous operator such that $(B \boldsymbol{\tau}, \mathbf{v})=b(\boldsymbol{\tau}, \mathbf{v})$. Then we see

$$
\begin{aligned}
\operatorname{ker} B & =\{\boldsymbol{\tau} \in \mathbf{L}: b(\boldsymbol{\tau}, \mathbf{v})=0 \quad \forall \mathbf{v} \in \mathbf{V}\} \\
& =\left\{\boldsymbol{\tau} \in \mathbf{L}:(\boldsymbol{\tau}, \nabla \mathbf{v})_{\Omega}=0 \quad \forall \mathbf{v} \in \mathbf{V}\right\} \\
& =\{\boldsymbol{\tau} \in \mathbf{L}: \operatorname{div} \boldsymbol{\tau}=0\} .
\end{aligned}
$$

It shows that $\tau \in \operatorname{ker} B$ is divergence free and hence Lemma 2.2 implies

$$
\|\boldsymbol{\tau}\|_{0}^{2} \lesssim\|\mathcal{A} \boldsymbol{\tau}\|_{0}^{2}=\nu a(\boldsymbol{\tau}, \boldsymbol{\tau}), \forall \boldsymbol{\tau} \in \operatorname{ker} B
$$

Now the inf-sup condition (8) and coercivity condition (9) imply that the variational problem (7) has a unique solution thanks to the theory of saddle point problems (see [10], [19]).

Remark 2.4. Theorem 2.3 implies the solution $\boldsymbol{\sigma}$ belongs to $\left[L^{2}(\Omega)\right]^{2 \times 2}$. Indeed, it follows from the second equation of (7) that $\boldsymbol{\sigma}$ admits weak divergence and $\operatorname{div} \sigma=-\mathbf{f}$ in $\Omega$.

\section{Nonconforming mixed finite element methods}

In this section, we shall consider finite dimensional approximation of our primal mixed variational formulation given by (7). For this, we consider nonconforming mixed finite elements. Let $\left\{\mathfrak{T}_{h}\right\}$ be a family of quasi-uniform triangulations of $\bar{\Omega}$ by triangles $T$ of diameter $h_{T}$. For triangle $T_{j} \in \mathfrak{T}_{h}$, let

$$
\Gamma=\partial \Omega, \quad \Gamma_{j}=\Gamma \cap \partial T_{j}, \quad e_{j k}=e_{k j}=\partial T_{j} \cap \partial T_{k} .
$$

Also, we define $\mathcal{E}_{h}$ to be the set of all edges of $\mathfrak{T}_{h}$. Given $T \in \mathfrak{T}_{h}$, we let $\mathcal{E}(T)$ be the set of its edges, and let $\mathcal{E}(\Omega)$ be the set of all boundary edges of $\Omega$, that is,

$$
\mathcal{E}(\Omega)=\left\{e \in \mathcal{E}_{h}: e \in \mathcal{E}(T) \cap \Gamma, \quad T \in \mathfrak{T}_{h}\right\} .
$$

In Section 2, we reviewed a primal mixed finite element method for continuous elliptic problems. To formulate a primal mixed version of discrete problem by using nonconforming finite elements, we need a finite element approximation to the space $\mathbf{L}$ and $\mathbf{V}$. Define

$$
\mathbf{L}_{h}=\left\{\boldsymbol{\tau} \in \mathbf{L}:\left.\boldsymbol{\tau}\right|_{T} \in\left(\mathcal{P}_{0}(T)\right)^{2 \times 2}, \int_{\Omega} \operatorname{tr} \boldsymbol{\tau}=0\right\}
$$


Notice that the space $\mathbf{L}_{h}$ inherits the trace free constraint from the space $\mathbf{L}$. And define the space $\mathbf{V}_{h}$ as follows:

$$
\begin{gathered}
\mathbf{V}_{h}=\left\{\mathbf{v} \in \mathbf{V}:\left.\mathbf{v}\right|_{T} \in\left(\mathcal{P}_{1}(T)\right)^{2}, N_{j k}(\mathbf{v})=N_{k j}(\mathbf{v}) \text { for all } j \neq k,\right. \\
\left.N_{j}(\mathbf{v})=0 \text { on } \Gamma_{j} \subset \Gamma\right\},
\end{gathered}
$$

where $N_{j}(\mathbf{v})$ and $N_{j k}(\mathbf{v})$ are the values of $\mathbf{v}$ at the midpoint of boundary edge $\Gamma_{j}$ and interior edge $e_{j k}$, respectively. And $\mathcal{P}_{l}(T)$ is the set of polynomials of total degree $l$ on element $T . \mathbf{V}_{h}$ is indeed the Crouzeix-Raviart finite element space [17]. Note that degrees of freedom for Crouzeix-Raviart spaces can be given by

$$
\frac{1}{\operatorname{meas}(e)} \int_{e} \mathbf{v} d s \forall \mathbf{v} \in \mathbf{V}, \forall e \in \mathcal{E}_{h} .
$$

Also, let $B_{h}$ be an operator on $\mathbf{L}_{h}$ corresponding to $B$ and

$$
\operatorname{ker} B_{h}=\left\{\boldsymbol{\tau}_{h} \in \mathbf{L}_{h}: \sum_{T}\left(\boldsymbol{\tau}_{h}, \nabla \mathbf{v}\right)_{T}=0 \quad \forall \mathbf{v} \in \mathbf{V}_{h}\right\}
$$

Remark 3.1. If $\boldsymbol{\tau}_{h} \in \operatorname{ker} B_{h}$, it is indeed divergence free and satisfies the normal flux continuity as in the continuous case. To see this, consider each edge $e$ in $\mathcal{E}_{h}$ shared by two elements $T$ and $T^{\prime}$ of $\boldsymbol{T}_{h}$. Denote by $\boldsymbol{\tau}_{T}$ the value of the function $\boldsymbol{\tau}_{h}$ restricted to the triangle $T$ and denote the jump of normal flux by $\llbracket \boldsymbol{\tau}_{\boldsymbol{h}} \rrbracket:=\boldsymbol{\tau}_{T} \cdot \mathbf{n}+\boldsymbol{\tau}_{T^{\prime}} \cdot \mathbf{n}^{\prime}$. Take a test function $\mathbf{v}$ in (13) as Crouzeix-Raviart basis function correspoding to the edge $e$. Then, by the choice of degrees of freedom and integration by parts, we easily see that

$$
\llbracket \tau_{\boldsymbol{h}} \rrbracket=0 \quad \text { on edge } e,
$$

which implies that

$$
\operatorname{ker} B_{h} \subset \operatorname{ker} B \text {. }
$$

With (10) and (11), we arrive at the nonconforming discretization of primal mixed formulation of the equation (7): Find $\left\{\boldsymbol{\sigma}_{h}, \mathbf{u}_{h}\right\} \in \mathbf{L}_{h} \times \mathbf{V}_{h}$ such that

$$
\begin{aligned}
\left(\kappa \mathcal{A} \boldsymbol{\sigma}_{h}, \boldsymbol{\tau}\right)_{\Omega}-\sum_{T}\left(\nabla \mathbf{u}_{h}, \boldsymbol{\tau}\right)_{T}=0 \quad \forall \boldsymbol{\tau} \in \mathbf{L}_{h}, \\
\sum_{T}\left(\boldsymbol{\sigma}_{h}, \nabla \mathbf{v}\right)_{T}=(\mathbf{f}, \mathbf{v})_{\Omega} \quad \forall \mathbf{v} \in \mathbf{V}_{h} .
\end{aligned}
$$

We will find the following lemma useful for convergence analysis.

Lemma 3.2. For any $\mathbf{w}_{h} \in \mathbf{V}_{h}$, we have the following two properties:

$$
\begin{aligned}
\int_{\Omega} \nabla_{h} \mathbf{w}_{h} d x & =0, \\
\int_{\Omega} \operatorname{tr}\left(\nabla_{\mathrm{h}} \mathbf{w}_{\mathrm{h}}\right) \mathrm{dx} & =0,
\end{aligned}
$$

where $\nabla_{h}$ denotes the piecewise gradient. 
Proof. The first equation (16) follows from integration by parts. Indeed, we have

$$
\begin{aligned}
\int_{\Omega} \nabla_{h} \mathbf{w}_{h} d x & =\sum_{T \in \mathfrak{T}} \int_{\mathcal{E}(T)} \mathbf{w}_{h} \otimes \mathbf{n} d s \\
& =\sum_{T \in \mathfrak{T}}\left\{\sum_{e \in \mathcal{E}(\Omega)} \int_{e} \mathbf{w}_{h} \otimes \mathbf{n} d s+\sum_{e \in \mathcal{E}_{h} \backslash \mathcal{E}(\Omega)} \int_{e} \mathbf{w}_{h} \otimes \mathbf{n} d s\right\} \\
& =0,
\end{aligned}
$$

where $\mathbf{n}$ is the outer unit normal vector and $\mathbf{w} \otimes \mathbf{n}$ denotes the tensor product whose $i j-$ th component is $w_{i} n_{j}$. The last equality follows from the choice of degrees of freedom for the finite element space $\mathbf{V}_{h}$ and the zero boundary condition. Next, the second equation (17) follows because the trace operator is linear and bounded.

Theorem 3.3. The discrete system (14)-(15) has a unique solution.

Proof. To prove the uniqueness of the solution it is enough to show that $\boldsymbol{\sigma}_{h}=0$ and $\mathbf{u}_{h}=0$ when $\mathbf{f}=0$. Assuming $\mathbf{f}=0$ in (15) implies that $\boldsymbol{\sigma}_{h} \in \operatorname{ker} B_{h}$. Taking $\boldsymbol{\tau}=\boldsymbol{\sigma}_{h}$ and $\mathbf{v}=\mathbf{u}_{h}$ leads to $\left(\kappa \mathcal{A} \boldsymbol{\sigma}_{h}, \boldsymbol{\sigma}_{h}\right)=0$ and so $\mathcal{A} \boldsymbol{\sigma}_{h}=0$. It follows from Lemma 2.2 and Remark 3.1 that $\boldsymbol{\sigma}_{h}=0$ because $\boldsymbol{\sigma}_{h} \in \operatorname{ker} B_{h}$ is divergence free. Next, by taking $\boldsymbol{\tau}=\nabla \mathbf{u}_{h}$ in (14), we easily see that $\mathbf{u}_{h}=0$ thanks to edge midpoint continuity and the homogeneous boundary condition.

In the next section, we derive the error estimate for the above finite element approximation.

\section{Error estimates}

For the error analysis of nonconforming finite element methods, it is convenient to use mesh-dependent norms and seminorms:

$$
\|\mathbf{v}\|_{1, h}:=\left(\sum_{T}|\mathbf{v}|_{1, T}^{2}\right)^{1 / 2} \text { for } T \in \mathfrak{T}_{h} .
$$

Notice that the nonconforming finite element space $\mathbf{V}_{h}$ contains a conforming finite element subspace $\mathbf{X}_{h} \subset \mathbf{V}$ of piecewise linear polynomials. We suppose that the following assumptions on the conforming subspaces $\mathbf{X}_{h} \subset \mathbf{V}$ and $\mathbf{Y}_{h} \subset$ $\left[L^{2}(\Omega)\right]^{2 \times 2}$ of piecewise polynomials of degree 1 and degree 0 , respectively, are fulfilled:

$[H 1]$ There is an interpolant $P:\left[H^{2}(\Omega)\right]^{2} \rightarrow \mathbf{X}_{h} \subset \mathbf{V}_{h}$ such that for $l$, with $0 \leq l \leq 1$ the estimates

$$
\begin{aligned}
|\mathbf{v}-P \mathbf{v}|_{l, T} & \lesssim h_{T}^{2-l}|\mathbf{v}|_{2, T} \quad \forall \mathbf{v} \in\left[H^{2}(T)\right]^{2}, \\
|| \mathbf{v}-\left.P \mathbf{v}\right|_{0, e} & \lesssim h_{e}^{3 / 2}|\mathbf{v}|_{2, T} \quad \forall \mathbf{v} \in\left[H^{2}(T)\right]^{2} .
\end{aligned}
$$

$[H 2]$ There is a projection $\Pi:\left[H^{1}(T)\right]^{2 \times 2} \rightarrow \mathbf{L}_{h}$ satisfying the estimate

$$
\| \boldsymbol{\tau}-\left.\Pi \boldsymbol{\tau}\right|_{0, T} \lesssim h_{T}|\boldsymbol{\tau}|_{1, T} \quad \forall \boldsymbol{\tau} \in\left[H^{1}(T)\right]^{2 \times 2} .
$$


Note that the Clement interpolant is an example satisfying $[H 1]$ (see [16]). Next, let $\widetilde{\Pi}$ be an $L^{2}(\Omega)$-orthogonal projection onto $\mathbf{Y}_{h}$. If we define

$$
\Pi \tau:=\widetilde{\Pi} \tau-\frac{1}{2|\Omega|} \int_{\Omega} \operatorname{tr}(\widetilde{\Pi} \tau) I,
$$

then $\Pi \tau$ satisfies the trace-free condition and the assumption [H2] holds.

We note that $\Pi \boldsymbol{\sigma}-\boldsymbol{\sigma}_{h}$ does not belong to $\operatorname{ker} B_{h}$ and we have difficulty in applying the saddle point theory in the convergence analysis. To obtain an element of ker $B_{h}$, we need to add an appropriate function to it. Similar idea has been applied, for example, to study unsteady Darcy flows in porous media [5]. Here we present a modified proof to fit in the trace-free pseudostress-velocity formulation.

Lemma 4.1. Let the exact solution $\boldsymbol{\sigma}$ belong to $V$. Then there exists a function $\chi_{h}$, orthogonal to ker $B_{h} \subset \mathbf{L}_{h}$, such that $\Pi \boldsymbol{\sigma}-\boldsymbol{\sigma}_{h}+\chi_{h}$ belong to ker $B_{h}$ and

$$
\left\|\chi_{h}\right\|_{0, \Omega} \lesssim h\|\sigma\|_{1, \Omega}
$$

Proof. Consider the following auxiliary problem of finding $\chi_{h} \in \mathbf{L}_{h}$ such that

$$
b\left(\chi_{h}, \mathbf{v}_{h}\right)=b\left(\boldsymbol{\sigma}_{h}-\Pi \boldsymbol{\sigma}, \mathbf{v}_{h}\right) \quad \forall \mathbf{v}_{h} \in \mathbf{V}_{h} .
$$

The inf-sup theory guarantees a unique existence of such function $\chi_{h} \in\left(\operatorname{ker} B_{h}\right)^{\perp}$ $\subset \mathbf{L}_{h}$ so that $\chi_{h}=\nabla \mathbf{w}_{h}$ in each $T$ for some $\mathbf{w}_{h} \in \mathbf{V}_{h}$ and $\chi_{h}$ is trace-free. Moreover, we have

$$
\left\|\chi_{h}\right\|_{0, \Omega} \leq \sup _{\mathbf{v}_{h} \in \mathbf{V}_{h}} \frac{b\left(\chi_{h}, \mathbf{v}_{h}\right)}{\left\|\mathbf{v}_{h}\right\|_{1, h}}=\sup _{\mathbf{v}_{h} \in \mathbf{V}_{h}} \frac{b\left(\boldsymbol{\sigma}_{h}-\Pi \boldsymbol{\sigma}, \mathbf{v}_{h}\right)}{\left\|\mathbf{v}_{h}\right\|_{1, h}}
$$

To estimate the right-hand side, consider each edge $e$ in $\mathcal{E}_{h}$ shared by two elements $T$ and $T^{\prime}$ of $\mathfrak{T}_{h}$. Denoting by $\overline{\mathbf{v}}_{e}$ the common mean value of the function $\left.\mathbf{v}_{h}\right|_{T}$ and $\left.\mathbf{v}_{h}\right|_{T^{\prime}}$ on $e$, and by $\overline{\boldsymbol{\sigma}}_{e} \cdot \mathbf{n}$ the mean value of $\boldsymbol{\sigma} \cdot \mathbf{n}$. Then, it follows from the orthogonality of the projection $\Pi$, (15) and Remark 2.4 that

$$
\begin{aligned}
b\left(\Pi \boldsymbol{\sigma}-\boldsymbol{\sigma}_{h}, \mathbf{v}_{h}\right) & =b\left(\boldsymbol{\sigma}-\boldsymbol{\sigma}_{h}, \mathbf{v}_{h}\right) \\
& =\sum_{T \in \mathfrak{T}_{h}}\left\{\left(\boldsymbol{\sigma}, \nabla \mathbf{v}_{h}\right)_{T}-\left(\mathbf{f}, \mathbf{v}_{h}\right)_{T}\right\} \\
& =\sum_{T \in \mathfrak{T}_{h}}\left\{-\left(\operatorname{div} \boldsymbol{\sigma}+\mathbf{f}, \mathbf{v}_{h}\right)_{T}+\int_{\partial T}(\boldsymbol{\sigma} \cdot \mathbf{n}) \mathbf{v}_{h} d s\right\} \\
& =\sum_{e \in \mathcal{E}_{h}} \int_{e}(\boldsymbol{\sigma} \cdot \mathbf{n}) \llbracket \mathbf{v}_{h} \rrbracket d s \\
& =\sum_{e \in \mathcal{E}_{h}}\left(\int_{e}\left(\boldsymbol{\sigma} \cdot \mathbf{n}-\overline{\boldsymbol{\sigma}}_{e} \cdot \mathbf{n}\right)\left(\left.\mathbf{v}_{h}\right|_{T}-\overline{\mathbf{v}}_{e}\right) d s\right. \\
& \left.+\int_{e}\left(\boldsymbol{\sigma} \cdot \mathbf{n}^{\prime}-\overline{\boldsymbol{\sigma}}_{e} \cdot \mathbf{n}^{\prime}\right)\left(\left.\mathbf{v}_{h}\right|_{T^{\prime}}-\overline{\mathbf{v}}_{e}\right) d s\right)
\end{aligned}
$$

where $\llbracket \mathbf{v}_{h} \rrbracket$ denotes the jump of $\mathbf{v}_{h}$ on edge $e$. 
By using the affine transformation that maps a reference element $\hat{T}$ onto $T$ and a generalized Bramble-Hilbert lemma, we have that

$$
\begin{aligned}
\left|\int_{e}\left(\boldsymbol{\sigma} \cdot \mathbf{n}-\overline{\boldsymbol{\sigma}}_{e} \cdot \mathbf{n}\right)\left(\left.\mathbf{v}_{h}\right|_{T}-\overline{\mathbf{v}}_{e}\right) d s\right| & \lesssim h\left\|\hat{\boldsymbol{\sigma}} \cdot \mathbf{n}-\overline{\boldsymbol{\sigma}}_{e} \cdot \mathbf{n}\right\|_{1, \hat{T}}\left\|\hat{\mathbf{v}}_{h}-\overline{\mathbf{v}}_{e}\right\|_{1, \hat{T}} \\
& \lesssim h|\hat{\boldsymbol{\sigma}} \cdot \mathbf{n}|_{1, \hat{T}}\left|\hat{\mathbf{v}}_{h}\right|_{1, \hat{T}} \\
& \lesssim h\|\boldsymbol{\sigma}\|_{1, T}\left\|\mathbf{v}_{h}\right\|_{1, T} .
\end{aligned}
$$

In the last inequality we use the switching back to $T$ argument. A similar calculation on $T^{\prime}$ and summing these estimates on $e$ then complete the proof of (21).

Now we are in a position to prove the main theorem of this section.

Theorem 4.2. Let the exact solution $\boldsymbol{\sigma}$ and $\mathbf{u}$ belong to $\left[H^{1}(\Omega)\right]^{2 \times 2}$ and $\left[H_{0}^{1}(\Omega)\right]^{2} \cap\left[H^{2}(\Omega)\right]^{2}$, respectively. Then, the error estimates for the method (14) and (15) hold:

$$
\begin{aligned}
\left\|\boldsymbol{\sigma}-\boldsymbol{\sigma}_{h}\right\|_{0, h} & \lesssim h|\boldsymbol{\sigma}|_{1, \Omega} \\
\left\|\mathbf{u}-\mathbf{u}_{h}\right\|_{1, h} & \lesssim h\left(|\mathbf{u}|_{2, \Omega}+\|\boldsymbol{\sigma}\|_{1, \Omega}\right) .
\end{aligned}
$$

Proof. First, we start with estimating $\left\|\boldsymbol{\sigma}-\boldsymbol{\sigma}_{h}\right\|$. Using the projection operator $\Pi$ defined in $(20)$, we have

$$
\left\|\boldsymbol{\sigma}-\boldsymbol{\sigma}_{h}\right\|_{0, T} \leq\left\|\Pi \boldsymbol{\sigma}-\boldsymbol{\sigma}_{h}\right\|_{0, T}+\|\boldsymbol{\sigma}-\Pi \boldsymbol{\sigma}\|_{0, T} .
$$

From Lemma 4.1, we can choose a function $\chi_{h} \in\left(\text { ker } B_{h}\right)^{\perp}$ such that $\Pi \sigma-$ $\boldsymbol{\sigma}_{h}+\chi_{h} \in \operatorname{ker} B_{h}$. It follows that

$$
\left\|\Pi \boldsymbol{\sigma}-\boldsymbol{\sigma}_{h}\right\|_{0, \Omega} \leq\left\|\Pi \boldsymbol{\sigma}-\boldsymbol{\sigma}_{h}+\chi_{h}\right\|_{0, \Omega}+\left\|-\chi_{h}\right\|_{0, \Omega}
$$

and the first term in the right-hand side can be estimated as follows: For simplicity, letting $\tilde{\boldsymbol{\sigma}}=\Pi \boldsymbol{\sigma}-\boldsymbol{\sigma}_{h}+\chi_{h}$, we have

$$
\begin{aligned}
\left\|\Pi \boldsymbol{\sigma}-\boldsymbol{\sigma}_{h}+\chi_{h}\right\|_{0, \Omega}^{2} & \lesssim\left\|\mathcal{A}\left(\Pi \boldsymbol{\sigma}-\boldsymbol{\sigma}_{h}+\chi_{h}\right)\right\|_{0, \Omega}^{2} \\
& =\left(\mathcal{A}\left(\Pi \boldsymbol{\sigma}-\boldsymbol{\sigma}_{h}-\boldsymbol{\sigma}+\boldsymbol{\sigma}+\chi_{h}\right), \tilde{\boldsymbol{\sigma}}\right)_{\Omega} \\
& =\left(\mathcal{A}\left(\Pi \boldsymbol{\sigma}-\boldsymbol{\sigma}+\chi_{h}\right), \tilde{\boldsymbol{\sigma}}\right)_{\Omega}+\left(\mathcal{A}\left(\boldsymbol{\sigma}-\boldsymbol{\sigma}_{h}\right), \tilde{\boldsymbol{\sigma}}\right)_{\Omega} \\
& \leq\left\|\Pi \boldsymbol{\sigma}-\boldsymbol{\sigma}+\chi_{h}\right\|_{0, \Omega}\|\tilde{\boldsymbol{\sigma}}\|_{0, \Omega}+\sum_{T} \nu\left(\nabla\left(\mathbf{u}-\mathbf{u}_{h}\right), \tilde{\boldsymbol{\sigma}}\right)_{T} \\
& \leq\left(\|\Pi \boldsymbol{\sigma}-\boldsymbol{\sigma}\|_{0, \Omega}+\left\|\boldsymbol{\chi}_{h}\right\|_{0, \Omega}\right)\|\tilde{\boldsymbol{\sigma}}\|_{0, \Omega} \\
& \lesssim h\left(|\boldsymbol{\sigma}|_{1, \Omega}+\|\boldsymbol{\sigma}\|_{1, \Omega}\right)\|\tilde{\boldsymbol{\sigma}}\|_{0, \Omega} .
\end{aligned}
$$

Here, $\sum_{T}\left(\nabla\left(\mathbf{u}-\mathbf{u}_{h}\right), \tilde{\boldsymbol{\sigma}}\right)_{T}=0$ follows in view of the fact $\tilde{\boldsymbol{\sigma}} \in \operatorname{ker} B_{h} \subset \operatorname{ker} B$. Collecting the above estimates leads to the desired result for the pseudostress variable with Lemma 4.1.

Next, for the interpolation operator $P:\left[H^{2}(\Omega)\right]^{2} \rightarrow X_{h}$ defined in (18), we have

$$
\left\|\nabla\left(\mathbf{u}-\mathbf{u}_{h}\right)\right\|_{0, T} \leq\left\|\nabla\left(P \mathbf{u}-\mathbf{u}_{h}\right)\right\|_{0, T}+\|\nabla(\mathbf{u}-P \mathbf{u})\|_{0, T}
$$


To estimate the first term, take $\boldsymbol{\tau}=\nabla_{h} \boldsymbol{\omega}$ where $\boldsymbol{\omega}=P \mathbf{u}-\mathbf{u}_{h}$. Then $\int_{\Omega} \operatorname{tr} \boldsymbol{\tau}=0$ by Lemma 3.2 and we have

$$
\left\|\nabla_{h}\left(P \mathbf{u}-\mathbf{u}_{h}\right)\right\|_{0, \Omega} \leq \sup _{\boldsymbol{\tau} \in \mathbf{L}_{h}} \sum_{T} \frac{\left(\nabla\left(P \mathbf{u}-\mathbf{u}_{h}\right), \boldsymbol{\tau}\right)_{T}}{\|\boldsymbol{\tau}\|_{0, \Omega}}
$$

Now, it follows that

$$
\begin{aligned}
\sum_{T}\left(\nabla\left(P \mathbf{u}-\mathbf{u}_{h}\right), \boldsymbol{\tau}\right)_{T} & =\sum_{T}(\nabla(P \mathbf{u}-\mathbf{u}), \boldsymbol{\tau})_{T}+\sum_{T}\left(\nabla\left(\mathbf{u}-\mathbf{u}_{h}\right), \boldsymbol{\tau}\right)_{T} \\
& =(\nabla(P \mathbf{u}-\mathbf{u}), \boldsymbol{\tau})_{\Omega}+\left(\mathcal{A}\left(\boldsymbol{\sigma}-\boldsymbol{\sigma}_{h}\right), \boldsymbol{\tau}\right)_{\Omega} \\
& \leq\left(\|\nabla(P \mathbf{u}-\mathbf{u})\|_{0, \Omega}+\left\|\mathcal{A}\left(\boldsymbol{\sigma}-\boldsymbol{\sigma}_{h}\right)\right\|_{0, \Omega}\right)\|\boldsymbol{\tau}\|_{0, \Omega} \\
& \leq\left(\|\nabla(P \mathbf{u}-\mathbf{u})\|_{0, \Omega}+\left\|\boldsymbol{\sigma}-\boldsymbol{\sigma}_{h}\right\|_{0, \Omega}\right)\|\boldsymbol{\tau}\|_{0, \Omega} \\
& \lesssim\left(h|\mathbf{u}|_{2, \Omega}+h\|\boldsymbol{\sigma}\|_{1, \Omega}\right)\|\boldsymbol{\tau}\|_{0, \Omega},
\end{aligned}
$$

which completes the proof of the theorem.

\section{Numerical experiments}

In this section we demonstrate the numerical behavior of the proposed method (14) and (15) for some test examples. To compute numerical solutions of the Stokes equations in the domain $\Omega=(0,1) \times(0,1)$, we use the nonconforming $\left(P_{1}\right)^{2}$ finite elements for the velocity approximation and $\left(P_{0}\right)^{2 \times 2}$ elements for the stress approximation. All computations were written in MATLAB and errors were measured in the $L_{2}$ norm.

Example 1 (Smooth polynomial solution). The right-hand side and the boundary conditions for the Stokes problem are chosen such that

$$
\begin{aligned}
& \mathbf{u}(x, y)=\left(x^{2}(1-x)^{2} y(1-y)(1-2 y),-y^{2}(1-y)^{2} x(1-x)(1-2 x)\right) \\
& p(x, y)=y-x
\end{aligned}
$$

is the exact solution.

In Tables 1 and 2 we display various errors for stress, pressure, and velocity where $h$ denotes the mesh size.

TABLE 1. Convergence rates of $\boldsymbol{\sigma}$ and $p$ for Example 1

\begin{tabular}{c||c|c||c|c}
\hline $1 / h$ & $\left\|\boldsymbol{\sigma}-\boldsymbol{\sigma}_{h}\right\|_{0, \Omega}$ & convergence order & $\left\|p-p_{h}\right\|_{0, \Omega}$ & convergence order \\
\hline \hline 4 & 0.1076 & $\cdot$ & 0.0652 & $\cdot$ \\
8 & 0.0530 & 1.0205 & 0.0311 & 1.0705 \\
16 & 0.0262 & 1.0173 & 0.0151 & 1.0421 \\
32 & 0.0130 & 1.0091 & 0.0151 & 1.0421 \\
\hline
\end{tabular}


TABLE 2. Convergence rates of $\nabla \mathbf{u}$ and $\mathbf{u}$ for Example 1

\begin{tabular}{c||c|c||c|c}
\hline $1 / h$ & $\left\|\nabla \mathbf{u}-\nabla \mathbf{u}_{h}\right\|_{0, \Omega}$ & convergence order & $\left\|\mathbf{u}-\mathbf{u}_{h}\right\|_{0, \Omega}$ & convergence order \\
\hline \hline 4 & 0.0553 & $\cdot$ & 0.0042 & \\
8 & 0.0297 & 0.8974 & 0.0012 & 1.8232 \\
16 & 0.0152 & 0.9657 & 0.0003 & 1.9376 \\
32 & 0.0077 & 0.9888 & 0.0001 & 1.9792 \\
\hline
\end{tabular}

Each table confirms that the convergence order of $\boldsymbol{\sigma}, p$, and $\nabla \mathbf{u}$ converges 1 with the $L_{2}$ norm as proved in Section 3. The right side of Table 2 suggests that the velocity error measured in the $L_{2}$ norm is of the second order as expected.

Example 2 (Trigonometric smooth function). Consider the Stokes equations with the exact solution given by

$$
\begin{aligned}
& \mathbf{u}(x, y)=\left(\sin \pi x^{2} \sin 2 \pi y,-\sin \pi y^{2} \sin 2 \pi x\right) \\
& p(x, y)=\cos \pi x \cos \pi y .
\end{aligned}
$$

As we see from Tables 3 and 4, we have similar results as in Example 1 for the trigonometric function.

TABLE 3. Convergence rates of $\boldsymbol{\sigma}$ and $p$ for Example 2

\begin{tabular}{c||c|c||c|c}
\hline $1 / h$ & $\left\|\boldsymbol{\sigma}-\boldsymbol{\sigma}_{h}\right\|_{0, \Omega}$ & convergence order & $\left\|p-p_{h}\right\|_{0, \Omega}$ & convergence order \\
\hline \hline 4 & 2.5143 & $\cdot$ & 0.6498 &. \\
8 & 1.2460 & 1.0128 & 0.2847 & 1.1906 \\
16 & 0.6209 & 1.0049 & 0.1340 & 1.0872 \\
32 & 0.3101 & 1.0017 & 0.0656 & 1.0308 \\
\hline
\end{tabular}

TABLE 4. Convergence rates of $\nabla \mathbf{u}$ and $\mathbf{u}$ for Example 2

\begin{tabular}{c||c|c||c|c}
\hline $1 / h$ & $\left\|\nabla \mathbf{u}-\nabla \mathbf{u}_{h}\right\|_{0, \Omega}$ & convergence order & $\left\|\mathbf{u}-\mathbf{u}_{h}\right\|_{0, \Omega}$ & convergence order \\
\hline \hline 4 & 2.3403 &. & 0.0836 &. \\
8 & 1.1792 & 0.9889 & 0.0214 & 1.9634 \\
16 & 0.5913 & 0.9958 & 0.0055 & 1.9620 \\
32 & 0.2959 & 0.9988 & 0.0014 & 1.9858 \\
\hline
\end{tabular}

Example 3 (Lid-driven cavity flow). Consider the lid-driven cavity flow problem in a square cavity $[-1,1] \times[-1,1]$ with the lid moving from left to right. We impose leaky boundary conditions, that is, $\left\{y=1 ;-1 \leq x \leq 1 \mid u_{1}=1\right\}$. 
The incompressibility condition implies that there exists a scalar stream function $\psi: \Omega \rightarrow \mathbb{R}$, uniquely defined up to an additive constant, such that

$$
u_{1}=\frac{\partial \psi}{\partial y}, \quad u_{2}=-\frac{\partial \psi}{\partial x} .
$$

We deduce that

$$
-\nabla^{2} \psi=\frac{\partial u_{2}}{\partial x}-\frac{\partial u_{1}}{\partial y}=\omega
$$

where $\omega$ is the two-dimensional (scalar) vorticity variable that acts in a direction orthogonal to the $x y$ plane. In order to illustrate the Moffatt eddies (small counter-rotating recirculations) in the bottom corners, we plot exponentially spaced streamlines in Figure 1 (right) as in [22].

As it can be seen in Figure 1 (left), the Stokes driven-cavity flow pressure solution is anti-symmetric about the vertical centerline and pressure is zero on this centerline.
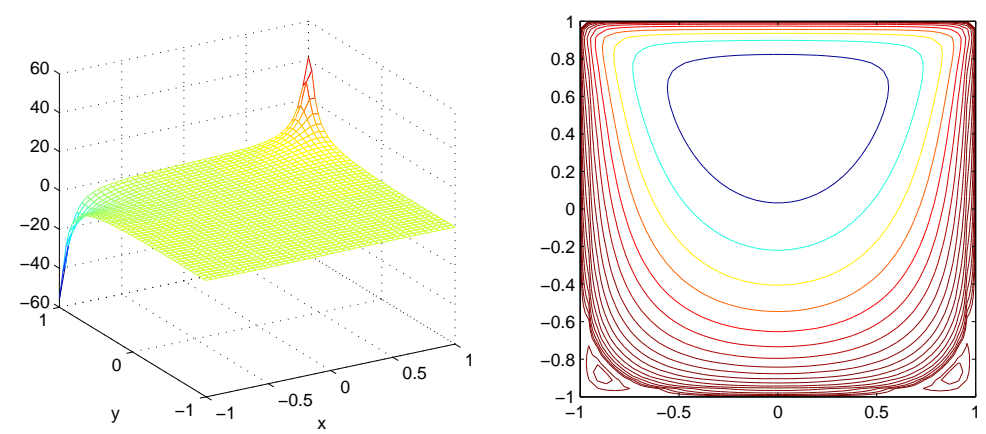

FiguRE 1. Pressure plot (left) and exponentially distributed streamlines (right)

\section{References}

[1] D. N. Arnold and F. Brezzi, Mixed and nonconforming finite element methods: implementation, postprocessing and error estimates, RAIRO Modél. Math. Anal. Numer. 19 (1985), no. 1, 7-32.

[2] D. N. Arnold and R. S. Falk, A new mixed formulation for elasticity, Numer. Math. 53 (1988), no. 1-2, 13-30.

[3] G. R. Barrenechea and G. N. Gatica, A primal mixed formulation for exterior transmission problems in $\mathbf{R}^{2}$, Numer. Math. 88 (2001), no. 2, 237-253.

[4] G. R. Barrenechea, G. N. Gatica, and J.-M. Thomas, Primal mixed formulations for the coupling of FEM and BEM. I. Linear problems, Numer. Funct. Anal. Optim. 19 (1998), no. 1-2, 7-32.

[5] C. Bernardi, V. Girault, and K. R. Rajagopal, Discretization of an unsteady flow through porous solid modeled by Darcy's equations, Math. Models Methods Appl. Sci. 18 (2008), no. $12,2087-2123$. 
[6] D. Braess, Finite Elements, Cambridge University Press, 1997.

[7] _ Enhanced assumed strain elements and locking in membrane problems, Comput. Methods Appl. Mech. Engrg. 165 (1998), no. 1-4, 155-174.

[8] D. Braess, C. Carstensen, and B. D. Reddy, Uniform convergence and a posteriori error estimators for the enhanced strain finite element method, Numer. Math. 96 (2004), no. 3, 461-479.

[9] S. C. Brenner and L. R. Scott, The Mathematical Theory of Finite Element Methods, Second edition. Texts in Applied Mathematics, 15, Springer-Verlag, New York, 2002.

[10] F. Brezzi and M. Fortin, Mixed and Hybrid Finite Element Methods, Springer Series in Computational Mathematics, 15, Springer-Verlag, New York, 1991.

[11] F. Brezzi, L. D. Marini, and P. Pietra, Two-dimensional exponential fitting and applications to drift-diffusion models, SIAM J. Numer. Anal. 26 (1989), no. 6, 1342-1355

[12] Z. Cai, B. Lee, and P. Wang, Least-squares methods for incompressible Newtonian fluid flow: linear stationary problems, SIAM J. Numer. Anal. 42 (2004), no. 2, 843-859.

[13] Z. Cai and G. Starke, First-order system least squares for the stress-displacement formulation: linear elasticity, SIAM J. Numer. Anal. 41 (2003), no. 2, 715-730.

[14] Z. Chen and J. Douglas, Jr, Approximation of coefficients in hybrid and mixed methods for nonlinear parabolic problems, Mat. Apl. Comput. 10 (1991), no. 2, 137-160.

[15] P. G. Ciarlet, Finite Element Method for Elliptic Problems, Society for Industrial and Applied Mathematics (SIAM), Philadelphia, PA, 2002.

[16] P. Clement, Approximation by finite element functions using local regularization, RAIRO Analyse Numérique 9 (1975), no. R-2, 77-84.

[17] M. Crouzeix and P.-A. Raviart, Conforming and nonconforming finite element methods for solving the stationary Stokes equations, RAIRO Anal. Numér. 7 (1973), no. R-3, $33-75$.

[18] M. Gerritsma and T. Phillips, Compatible spectral approximations for the velocity-stresspressure formulation of the Stokes problem, SIAM J. Numer. Anal. 28 (1991), 591-623.

[19] V. Girault and P. A. Raviart, Finite Element Methods for Navier-Stokes Equations: theory and algorithms, Springer Series in Computational Mathematics 5, 1986.

[20] D. Kim and E.-J. Park, Primal mixed finite-element approximation of elliptic equations with gradient nonlinearities, Comput. Math. Appl. 51 (2006), no. 5, 793-804.

[21] _ A priori and a posteriori analysis of mixed finite element methods for nonlinear elliptic equations, SIAM J. Numer. Anal. 48 (2010), no. 3, 1186-1207.

[22] M. Norburn and D. Silvester, Stable vs. stabilised mixed methods for incompressible flow, Computer Methods in Applied Mechanics and Engineering 166 (1998), 131-141.

[23] J. Park, A primal mixed domain decomposition procedure based on the nonconforming streamline diffusion method, Appl. Numer. Math. 50 (2004), no. 2, 165-181.

[24] J. E. Roberts and J. M. Thomas, Mixed and Hybrid methods, Handbook of Numerical Analysis (P. G. Ciarlet and J. L. Lions, eds.) Vol II, 523-639, Finite Element Methods (Part 1), North-Holland, Amsterdam, 1989.

[25] J. C. Simo and M. S. Rifai, A class of mixed assumed strain methods and the method of incompatible modes, Internat. J. Numer. Methods Engrg. 29 (1990), no. 8, 1595-1638.

[26] S. T. Yeo and B. C. Lee, Equivalence between enhanced assumed strain method and assumed stress hybrid method based on the Hellinger-Reissner principle, Internat. J. Numer. Methods Engrg. 39 (1996), no. 18, 3083-3099.

Sungmin Cho

Department of Mathematics

YONSEI UNIVERSITY

Seoul 120-749, Korea

E-mail address: smin95@yonsei.ac.kr 
Eun-Jae PARK

Department of Mathematics and

Department of Computational Science and Engineering

YONSEI UNIVERSITY

SeOul 120-749, Korea

E-mail address: ejpark@yonsei.ac.kr 